\title{
Isolation Precautions for Hospitalized Patients: The Challenges of Identifying Unintended Individual Consequences and Measuring the Prevention of Community Harm
}

\author{
Jason E. Bowling, M.D. and Barbara S. Taylor, M.D., M.S.
}

University of Texas Health Science Center San Antonio, San Antonio, TX, USA.

J Gen Intern Med 32(3):238-40

DOI: $10.1007 / \mathrm{s} 11606-016-3926-5$

(c) Society of General Internal Medicine 2016

I solation of potentially infectious individuals has been an accepted and sometimes effective form of infection control since the Middle Ages, when coastal cities protected their populace from plagues through quarantine, and separate colonies were established for those with Hansen's disease (leprosy). Isolation for infection control has also been associated with adverse events such as stigma and lack of access to medical care. As with all public health interventions, the benefit to the public in the form of averted contagion must be balanced with the harm to the individual. In this issue of JGIM, Tran and colleagues present results from a multi-site retrospective cohort study examining the impact of respiratory and contact precautions on patient outcomes and cost of care. ${ }^{1}$ The risk to public health from healthcare-associated infection is real. The Centers for Disease Control and Prevention estimate that, on any given day, one in 25 hospital patients has at least one healthcareassociated infection, which leads to morbidity, mortality, extended hospital stays, and increased healthcare costs. ${ }^{2}$

Tran and colleagues examined multiple outcomes: 30-day readmissions, emergency department visits, hospital length of stay, expected length of stay, adverse events, in-hospital mortality, patient complaints, and cost of care in patients placed on contact isolation for methicillin-resistant Staphylococcus aureus (MRSA) or droplet isolation for those with fever and respiratory symptoms (cases) and control patients admitted to the general medical services of three academic hospitals in Toronto, Canada. Cases and controls were propensity score-matched, a technique meant to ensure that one group was not more clinically ill than the other. They found that length of stay, expected length of stay, and costs were increased for those isolated, but they did not detect any increase in adverse events, patient complaints, or mortality in isolated patients. Their findings shed light on the consequences of isolation for the individual patient, and also highlight the difficulty of designing studies to address gaps in our knowledge regarding the balance of public health benefit and individual risk for isolation precautions.

Published online November 18, 2016
Do isolation precautions increase individual risk or pose a patient safety concern? The majority of the published studies that attempt to answer this question have specifically evaluated patients placed in contact precautions. In one of the more highly cited published studies on this topic, researchers evaluated adverse events in patients placed in contact isolation for MRSA colonization or infection at two different hospitals. This was a retrospective medical record review that matched 150 patients hospitalized between 1999 and 2000 with two controls for each patient. ${ }^{3}$ Investigators demonstrated a significant difference in the rate of study-determined preventable events, which included falls, pressure ulcers, and electrolyte disorders, with 20 versus 3 adverse events per 1000 days $(p<0.001)$ in patients placed on contact precautions versus controls. No differences were noted in mortality between the two groups. Although patients were matched for severity of illness by accepted tools (i.e. Charlson comorbidity index, APACHE II score), the cases were a combination of patients colonized and infected with MRSA, which suggests some heterogeneity in risk factors for morbidity. Another confounding element was that patients in contact precautions had a much longer length of stay. This was statistically accounted for in the calculation of the rate of adverse events, but complicated the ability to interpret patient satisfaction results.

A comprehensive review of the literature performed to evaluate adverse outcomes associated with contact precautions identified 15 studies published between 1989 and 2008. ${ }^{4}$ During this review, the authors identified four main adverse outcomes: less patient-healthcare worker contact, changes in systems of care leading to more noninfectious adverse events, increased symptoms of depression and anxiety, and decreased patient satisfaction with care. They concluded that the available studies were variable in content and results. All studies were observational, and many contained methodological shortcomings. The authors also noted potential publication bias towards studies demonstrating negative outcomes. Another systematic review examining this issue also concluded that contact isolation may negatively impact patient care, but that well-validated tools should be used in larger studies for better assessment. ${ }^{5}$

To address concerns with currently published studies, a recent cluster-randomized trial evaluated the effect of the universal use of gloves and gowns in the intensive care unit 
(ICU) on the acquisition of antibiotic-resistant bacteria. Adverse events were examined as a secondary outcome. ${ }^{6}$ Researchers used the Institute for Healthcare Improvement's IHI Global Trigger tool to identify and record adverse events from 1800 randomly selected charts of adult patients not colonized with antibiotic-resistant bacteria across 20 hospital sites from January through October 2012. The rate of adverse events in the ICU, including preventable, non-preventable, severe, and not severe, was lower in the intervention group using universal gloves and gowns than the control arm, but not statistically significant (58.7 events vs. 74.4 events per 1000 patient days; $95 \% \mathrm{CI},-40.7$ to $9.2 ; p=0.24)$. This was the first study to investigate adverse effects in patients randomly assigned to universal gown and glove use. ${ }^{7}$ These results do not show increased risk for isolated patients, and offer a different conclusion from that of many previously published observational studies showing increased adverse events in isolated patients. It should be noted that this trial specifically evaluated patients in the ICU setting, which routinely has a higher nurse-topatient staffing ratio than the general ward settings in which several of the prior studies occurred.

Despite these systematic reviews and data from one randomized controlled trial, we lack definitive answers to several fundamental questions. Are isolation precautions harmful to the patient? Do isolation precautions actually protect other patients in the hospital from healthcare-associated infection? Further evaluation in well-designed trials is needed to answer such questions, and to determine whether they vary by pathogen, condition, or mechanism of isolation. The ideal study in a hospital setting would be a prospective randomized controlled trial: screen all patients on admission, and randomize them to isolation or non-isolation. Regular follow-up screening of patients, visitors, and staff would be required to determine whether subsequent in-hospital infections were actually healthcare-associated rather than imported by a visiting family member. Adverse events would need to be rigorously measured, as in the randomized controlled trial described above. ${ }^{6,7}$ Subsequent infections in the hospital would need to be genetically linked using phylogenetic analysis tools prior to assuming that they were the result of healthcare-associated transmission. Finally, standard outcomes should be used: mortality, costs, and length of stay are all accepted as clinically relevant. Other endpoints such as hospital readmission are too dependent on pre-existing conditions and patient social, financial, and structural support to be relied upon. Such a study would allow a true assessment of the risk to patients in the isolation arm versus the benefits to the community, by determining whether there was increased hospital-based transmission of infections in the non-isolation arm.

Yet, conducting the ideal study as described above is difficult, time-consuming, and costly. The current state of the literature on isolation precautions reflects the daunting task of investigation in this area. Patient harm and adverse effects can be readily categorized and quantified. In contrast, measuring the prevention of harm, particularly to other patients and healthcare providers, is complex and costly. While there is some variance in how frequently and to what conditions isolation precautions are applied, ${ }^{8}$ isolation is still considered a standard practice, and is generally agreed to be mandated for certain conditions. Randomizing certain patients to no isolation precautions would be highly controversial. There are also inherent difficulties in accommodating a study design to understand how adverse events may differ with different types of isolation precautions (e.g. contact, droplet, airborne) required for different pathogens and infections. Donning and doffing the necessary personal protective equipment (PPE) for droplet precautions is much less timeconsuming than the same process for contact precautions, which could account for significant variation in issues like compliance and healthcare worker room entry. This also highlights another challenge in study design: patient education about isolation precautions and PPE impacts patient satisfaction with care. Mandating appropriate standardized patient education, and perhaps the number of healthcare worker visits per hour per patient, might negate many of the potential associated adverse effects. Variation in this area of healthcare worker practice might exist among facilities, and would be expected to impact results of any multi-center trial. Considering these challenges, it is not surprising that the majority of studies examining isolation precautions are observational, and thus subject to unknown confounders and publication bias.

The numerous ethical, logistic, and financial barriers to carrying out the ideal prospective randomized controlled trial are daunting, but not impossible to overcome. Tools from implementation science can be used to allow rigorous measurement in real world contexts, where randomization is not possible. Stepped wedge designs could be utilized, where a series of interventions is rolled out over time, allowing assessment of the impact of each intervention. ${ }^{9}$ These would enable investigators to determine which of the many aspects of isolation actually have an impact, or which measures, such as mandating healthcare worker visits per hour per patient, might mitigate harm. Propensity-matched case-control studies, such as the one by Tran and colleagues in this issue, provide important data. Even simple pre- and post-intervention designs, where adverse events and outcomes are rigorously measured, can be useful. None of these methodologies eliminates the overarching problem, however, that it is far easier to measure individual harm than community benefit. Yet, as patients, providers, and hospital systems struggle under the burden of isolation policies, a clearer understanding of their risks and benefits must be sought. Though ships quarantined in the harbors of the Mediterranean in the fourteenth century could not demand randomized controlled trials, surely the twenty-first century can bring us better standards of 
evidence-based practice, and more studies such as the one presented here, to shed light on this issue.

Corresponding Author: Jason E. Bowling, M.D.; University of Texas Health Science Center San Antonio, 7703 Floyd Curl Drive, MSC 7881, San Antonio, TX 78229-3900, USA (e-mail: Bowling@uthscsa. edu).

\section{Compliance with Ethical Standards:}

Conflict of Interest: No relevant conflicts of interest.

\section{REFERENCES}

1. Tran K, Bell C, Stall N, et al. The effect of hospital isolation precautions on patient outcomes and cost of care: a multi-site, retrospective, propensity score-matched cohort study. J Gen Intern Med. 2016. doi:10.1007/ s11606-016-3862-4.
2. Centers for Disease Control and Prevention. 2014 National and State Healthcare-Associated Infections Progress Report. Published March, 2016. Available at www.cdc.gov/hai/surveillance/. Accessed 12 Oct 2016.

3. Stelfox HT, Bates DW, Redelmeier DA. Safety of patients isolated for infection control. JAMA. 2003;290(14): 1899-1905.

4. Morgan DJ, Diekema DJ, Sepkowitz K, Perencevich EN. Adverse outcomes associated with Contact Precautions: a review of the literature. Am J Infect Control. 2009;37(2):85-93.

5. Abad C, Fearday A, Safdar N. Adverse effects of isolation in hospitalised patients: a systematic review. J Hosp Infect. 2010;76(2):97-102.

6. Harris AD, Pineles L, Belton B, et al. Universal glove and gown use and acquisition of antibiotic-resistant bacteria in the ICU: a randomized trial. JAMA. 2013;310(15):1571-1580.

7. Croft LD, Harris AD, Pineles L, et al. The effect of universal glove and gown use on adverse events in intensive care unit patients. Clin Infect Dis. 2015;61(4):545-553.

8. Martin EM, Russell D, Rubin Z, et al. Elimination of routine contact precautions for endemic methicillin-resistant Staphylococcus aureus and vancomycin-resistant Enterococcus: a retrospective quasi-experimental study. Infect Control Hosp Epidemiol. 2016:1-8.

9. Brown CA, Lilford RJ. The stepped wedge trial design: a systematic review. BMC Med Res Methodol. 2006;6:54. 